\title{
NEOPLASTIC CONSTRICTIVE PERICARDIAL DISEASE CASE REPORT AND SUBJECT REVIEW
}

by M. Yusuf Khan, M.D.*

Robert P. Moore, M.D.+

\section{DOI: http://dx.doi.org/10.5915/14-3-12133 SUMMARY}

An unusual case of neoplastic restrictive pericardiomyopathy from metastatic constrictive pericardial disease from a primary cholangiocarcinoma of liver clinically mimicked alcoholic cardiomyopathy.

\section{INTRODUCTION}

Neoplastic constrictive pericardial disease is not a frequent occurrence and when present, usually results from metastases to the pericardium from carcinoma of the lung and gastrointestinal tract ${ }^{1},{ }^{2}, 3,4$. Recently, we observed a case of metastatic constrictive pericaditis from a primary cholangiocarcinoma of liver.

\section{CLINICAL SUMMARY}

A 53 year old black male was admitted to College Hospital with a chief complaint of progressive swelling of the feet and legs for eight months duration. He dinied abdominal swelling. Four months prior to admission, he started having dyspnea on exertion, at first after walking four to five blocks and later on walking even one block, but denied paroxysmal nocturnal dyspnea. He also complained of intermittent palpitations. His appetite had been poor and he had lost 30 pounds during the two months before admission. there was no history of diabetes mellitus or hypertension. Pulmonary tuberculosis had been diagnosed four years earlier, adequately treated for 18 months. He drank in moderation and did not smoke.

On admission, the patient was markedly cachectic and febrile. Pulse was 150 to the minute and regular; blood pressure was $90 / 60 \mathrm{~mm}$. $\mathrm{Hg}$. in both arms and respirations were 18 to the minute. There was marked jugular venous distension at 45 degrees. Carotid and other peripheral pulsations were feeble. Slight scleral icterus was noted. There was no lymphadenopathy. Examination of the cardiovascular system revealed the point of maximal impulse to be in the fifth intercostal space just outside the midclavicular line.. No heaves or thrills were felt. The SI was normal and of constant intensity. S2 was also normal. No gallops or murmurs were heard. Lung fields were clear to auscultation and percussion. Abdominal examination demonstrated an enlarged liver that measured $15 \times 19$ $\mathrm{cm}$. and was firm and tender. The was $4+$ pitting edema of lower extremities extending to the thighs. The neurologic examination was essentially normal. Laboratory investigations revealed hemoglobin of $14.9 \mathrm{G}$./ dl. with a hematocrit of 42.0 vols. $\%$; the white blood cells count was $12,700 \mathrm{~mm}^{3}$. with $95 \%$ segs, $2 \%$ lymphs and $3 \%$ monocyts. Blood chemistry revealed sodium of $126 \mathrm{mEq}$. / ., chloride $90 \mathrm{mEq}$. I, carbon dioxide $26 \mathrm{mEq}$. /., potassium $5.3 \mathrm{mEq} / \mathrm{I}$., BUN 16 $\mathrm{mg}$./dl. with a creatine of $1.0 \mathrm{mg} / \mathrm{dl}$., calcium 8.1 mg./ dl. and phosphate $2.9 \mathrm{mg}$./dl.; SGOT 236, SGPT 234 and LDH 420. Alkaline phosphatase was 240. Total serum billirubin was $2.8 \mathrm{mg}$. $\mathrm{dl}$. with a direct of $0.6 \mathrm{mg}$. $/ \mathrm{dl}$. Total serum proteins were $6.9 \mathrm{G}$. $/ \mathrm{dl}$., with albumin 4.3 and globulin $2.6 \mathrm{G}$./ dl. Initial blood gases revealed a $\mathrm{pH}$ of $7.35, \mathrm{PO} 2$ of $80.5, \mathrm{PCO} 2$ of 42 and $\mathrm{HCO} 3$ of 27.6. Electrocardiogram was consistent with atrial flutter with a ventricle response of 150 to the minute. A roengenogram of the chest showed cardiomegaly and a possible infiltrate in the left lower lobe obscuring the left heart border.

HOSPITAL COURSE: A preliminary diagnosis of cardiomyopathy secondary to alcohol was made. Upon arrival to the Intensive Care Unite, the patient was cardioverted with 50 joules of energy. The electrocardiogram following cardioversion showed low voltage, sinus tachycardia and right ventricular hypertrophy. The patient was started on Digoxin, Lasix and aqueous penicillin. Despite $200 \mathrm{mg}$. of Lasix in divided doses, the patient did not secrete any urine during the next 12 hours. A Swan-Ganz catheter was inserted via the brachial approach and the following pressures obtained: Right atrium (mean) 22; right

* Associate Professor of Pathology, Deparment of Pathology, CMDNJ-New Jersey Medical School, 100 Bergen Sireet, Newark. New Jersey 07103.

+ Resident in Pathology, Department of Pathology, CMDNJ - New Jersey Medical School, 100 Bergen

Street, Newark, New Jersey 07103.

Reprints, Requests: Dr. Khan, Department of Pathology. 
ventricle $65 / 22$; pulmonary artery $65 / 25$; PCW (mean) $25 \mathrm{~mm}$. $\mathrm{Hg}$. Cardiac output was measured to be 2.0 litres per minute per $\mathrm{M}^{2}$. The pressures were thought to be consistent with constrictive pericarditis. Percariectomy was performed on the fourth hospital day. A markedly thickened, fibrous and "calcified" pericardium was stripped off with difficulty during surger. Following surgery, the patient continued to do poorly. No decrease in jugular venous pressure was noted and the patient continued to have marked edema, sinus tachycardia, poor urine output, and expired few days after surgery. Pathological diagnosis of the excised pericardium was reported as metastatic carcinoma.

AUTOPSY FINDINGS: Significant autopsy findings were limited to heart, liver and prostate. Ventral portion of parietal pericardium was missing. The remaining pericardium, thickened by greyish-white tumor infiltrate, was firmly adnerent to the heart. The ventral visceral epicardial surface was studded diffusely by nodular carcinomatour implants that extended deep into left ventricular myocardium (Fig. 1). The mycardium of atria and right ventricular outflow tract, in particular, showed extensive tumor infiltration. The pericardial sleeve extending over aorta and pulmonary artery was massively infiltrated by greyish-white tumor encasing the superior vena cava, aorta and right and left pulmonary arteries. Superior vena cava and pulmonary arteries at the level of pulmonary trunk bifurcation were compressed and stenotic (Fig. 2) Right ventricle was moderately dilated. Pleura on both sides were irregularly thickened and lower lobes of both lungs were firmly adherent to diaphragmatic surfaces, more marked on right side. Both lungs showed multiple parenchymal metastic tumor nodules. The liver, at the interlobar zone, contained an infiltrating greysih-white, firm to hard tumor with ill-defined edges and multiple satellite nodules towards its periphery. Tumor involved one of the main branches of the left hepatic duct with evodent hepatic vein vascular invasion. The remaining part of the left lobe showed focal areas of centrolobular necrosis and grey-dark red mottling (Fig. 3). Histologically, the tumor in the liver was an infiltrating desmoplastic hepatic duct adenocarcinomacal intraductal papillary architecture and marked anaplasia. Extensive lymphatic and vascular invasion was present within and outside the liver with metastatic carcinomatosis of the diaphragm, pleura, lungs, pericardium and myocardium. Prostate contained an incidental focus of prostatic adeoncarcinoma.

\section{COMMENTS}

To our knowledge, this is the first case of symptomatic neoplastic constrictive pericardial and infiltrative myocardial disease from metastatic cholangiocarcinoma of liver. Peripheral edema as the presenting sign is of somewhat unusual occurrence in constrictive perdarditis. The combination of general pericardial constriction associated with marked constriction of main vessles and infiltrative myocardial disease may explain such a presentation in this case. The incidence of cardiac metastases from neoplastic diseases is extremely variable, raning from 0.1 to $21 \%$ of cancer autopsies $1,3,4,5$. The metastases are usually multiple and very rarely solitary. Malignancies most commonly associated with metastatic disease of heart are primary in the lungs and breast, melanomas and lymphomas, and leukemia ${ }^{36}$. Cardiac involvement in metastatic disease is typically characterized by involvement of pericardium, myocardium or both, and is manifested clinically by pericardial effusion, pericardial construction with or without effusion, and infiltrative cardiomyopathy with or without associated pericardial disease ${ }^{3}$ ?. Metastatic heart disease may be the primary cause of death, contribute to death or be unrelated to both. Most often, it is asymptomatic and discovered only as an incidental finding at autopsy. When symptomatic, antemortem diagnosis is made in only very small percentage of the cases and often missed ${ }^{3}$.

Pericardial involvement by metastatic neoplastic disease, although not uncommon, most commonly is an incidental finding at necropsy ${ }^{2}, 3,5,7$. In very small minority of cases it may, however, dominate the clinical picture. In such symptomatic cases, symptoms are caused by serosanquineous pericardial effusion with tamponade and only rarely by constrictive pericarditis or restrictive cardiomyopathy ${ }^{8}, 9$. Restrictive cardiomyopathy usually results from extensive tumor infiltration of myocardium. Very rarely, tumors may show direct intracavitary cardiac extension ${ }^{10}$. Cardiac tamponade may be the first manifestation of the metastatic malignant disease, usually develops slowly and is often missed ${ }^{3}$. Primary malignant tumors of the pericardium, although extremely uncommon, cause pericardial effusion and tamponade more readily than metastatic tumors. Metastatic involvement of the pericardium and myocardium, on the other hand, more frequently causes restrictive pericardiomyopathy. In patients with malignant disease, pericardiomyopathy may occasionally result from radiation therapy of tumors adjacent to heart, with or without metastatic involvement of pericardium or myocardium. ${ }^{6},{ }^{11},{ }^{12}$.

\section{REFERENCES}

I. Mohiuddin, A.B.: Constrictive Pericarditis. An analysis of 17 cases. Dis. Chest 51:298-303, 1967.

2. Eissa, M.H.; Khalil, A.M. and Kadry, S.M.: Second- 
ary Malignant Tumors of Heart and Pericardium. JOurnal of Egyptian Medical Association 58:337-349. 1975.

3. Theologides, Athanasias: Neoplastic Cardiac Tamponade. Seminars in Oncology 5: 181-192, 1978.

4. Takagi, Toshitaka: The Pericardium as Seen at Autopsy. Japanese Circulation Journal 42: 116-130. 1978.

5. Thurber, D.L.: Edwards, J.E.: Achor, R.W.P.: Secondary Malignant Tumors of the Heart and Pericardium. Circulation 26: 228-241. 1962.

6. Greenwood, Ronald D.; Rosenthal, Amon: Cassady, Robert: Jaffe. Norman and Nades. Alexander S.: Constrictive Pericarditis in Childhood Due to Mediastinal Irradiation. Circulation 50: 1033-1039. 1974.

7. Mann, Tift: Brodie, Bruce R :- Grossman, William and McLaurin, Lambert: Effusive-Constrictive Hemodynamic Pattern due to Neoplastic Involvement of the Pericardium. The American Joumal of Cardiology 41:

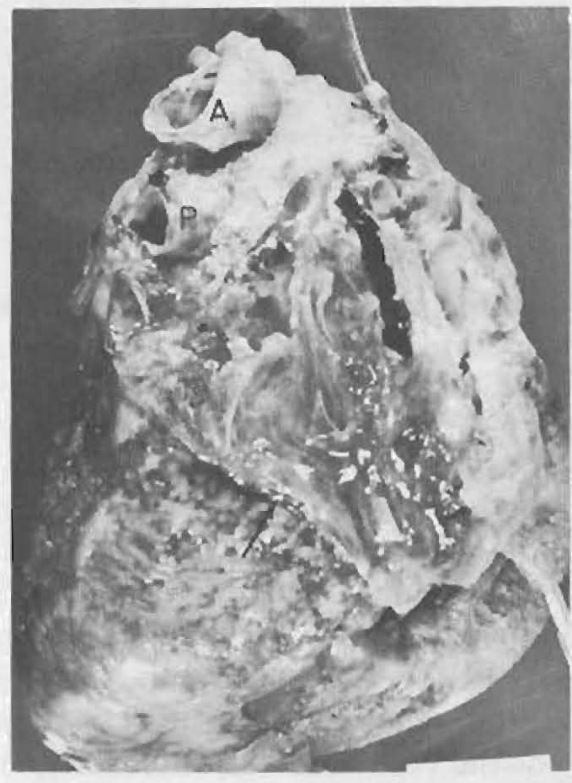

Figure 1

Heart showing partial pericardiectomy with extensive tumor metastases. Note the narrowing of aorta (A), pulmonary artery (P) by tumor. Probe passing through superior and inferior vena cava. Resected pericardial edge (arrow).
$781-786,1978$

8. Slater, S.R.; Kroop, 1.G.; Zuckerman, S.: Constrictive Pericarditis Caused by Solitary Metastic Carcinosis of the Pericardium and Complicated by Radiation Fibrosis of the Midiastinum. Am. Hearl J. 43:401-4I2, 1952.

9. Spodick, David H.; Sundershan, Kumar: Subacute Constrictive Pericarditis with Cardiac Tamponade. Dis. Chest 54: 62-66, 1968.

10. Ehrich, Dennis A.; Widmann, Jean J.; Berger, Robert L. and Abelman, Walter H.: Intracavitary Cardiac Extension of Hepatoma. The Annals of Thoracic Surgery 19: 206-211, 1975.

11. MacLeod. Cathel A.: Schwart7. Harold and Linton. Donald B.: Constrictive Pericarditis Following Irraditation Therapy. JAMA 207: 2281-2282, 1969.

12. Cohn, K.E.: Stewart. J.R.: Fajardo. L.F., et. al.: Heart Disease Following Radiation. Medicine 46: 281-298. 1967.

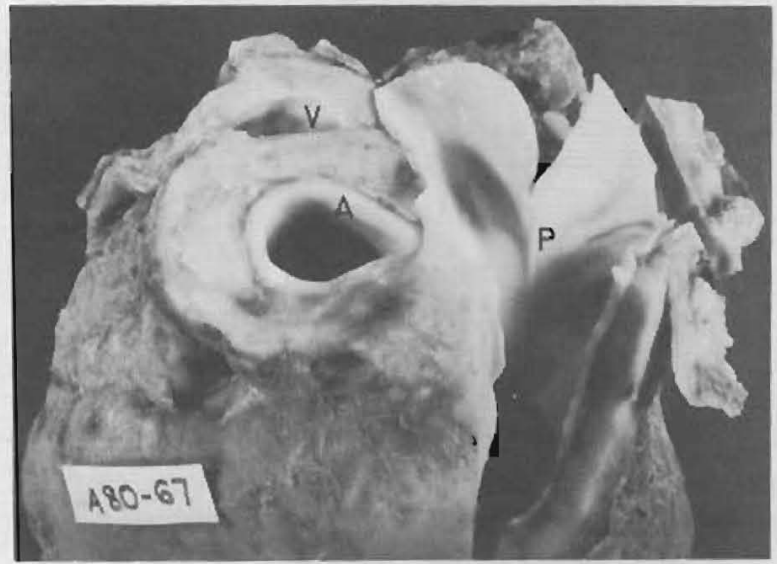

Figure 2

Note massive metastatic tumor infiltration of pericardium and pericardial space around aorta (A). pulmonary artery (P) and superior vena cava (V).

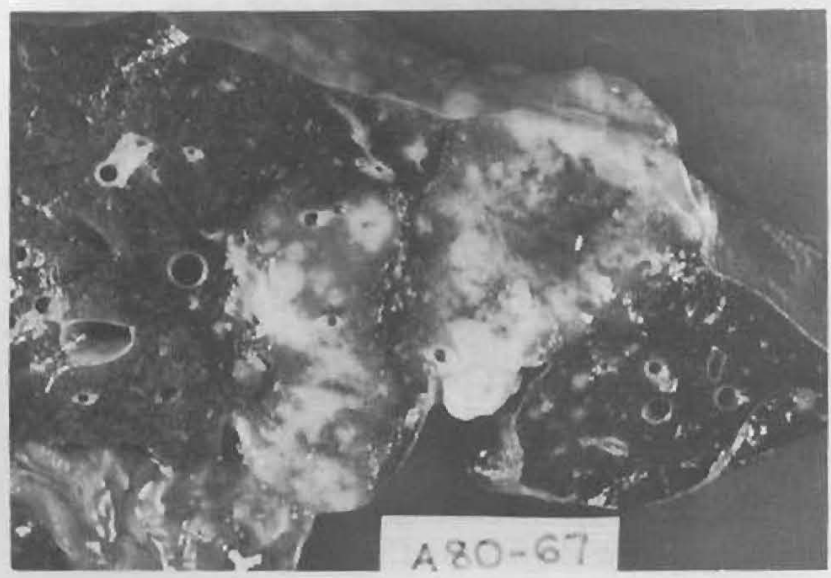

Figure 3

Liver showing primary infiltrating tumor mass in the interlobar zone. 